\title{
Transport of GenX in Saturated and Unsaturated Porous Media
}

\author{
Ni Yan ${ }^{1,2,3}$, Yifan $\mathrm{Ji}^{1}$, Bohan Zhang ${ }^{1}$, Xilai Zheng ${ }^{1}$, Mark L. Brusseau ${ }^{3, *}$ \\ ${ }^{1}$ Key Lab of Marine Environmental Science and Ecology, Ministry of Education, College of \\ Environmental Science and Engineering, Ocean University of China, Qingdao, 266100, P.R. \\ China; \\ ${ }^{2}$ MOE Key Laboratory of Groundwater Circulation and Environmental Evolution, China University \\ of Geosciences (Beijing), Beijing, 100083, P.R. China \\ ${ }^{3}$ Environmental Science Department, University of Arizona, Tucson, AZ 85721, United States
}

\section{Abstract}

The objective of this research was to investigate the retention and transport behavior of GenX in five natural porous media with similar median grain diameters, but different geochemical properties. Surface tensions were measured to characterize surface activity. Miscible-displacement experiments were conducted under saturated conditions to characterize the magnitude of solidphase adsorption, while unsaturated-flow experiments were conducted to examine the impact of air-water interfacial adsorption on retention and transport. The results from surface-tension measurements showed that the impact of solution composition is greater for the ammonium form of GenX than for the acid form, due to the presence of the $\mathrm{NH}_{4}$ counterion. The breakthrough curves for the experiments conducted under saturated conditions were asymmetrical, and a solutetransport model employing a two-domain representation of nonlinear, rate-limited sorption provided reasonable simulations of the measured data. The magnitudes of solid-phase adsorption were relatively small, with the highest adsorption associated with the medium containing the greatest amount of metal oxides. The breakthrough curves for the experiments conducted under unsaturated conditions exhibited greater retardation due to the impact of adsorption at the air-water interface. The contributions of air-water interfacial adsorption to GenX retention ranged from $\sim 24 \%$ to $\sim 100 \%$. The overall magnitudes of retardation were relatively low, with retardation factors $<\sim 3$, indicating that GenX has significant migration potential in soil and the vadose zone. To our knowledge, the results presented herein represent the first reported data for solid-water and air-water interfacial adsorption of GenX by soil. These data should prove useful for assessing the transport and fate behavior of GenX in soil and groundwater.

\section{Graphical Abstract}

\footnotetext{
“Corresponding author, Brusseau@email.arizona.edu.

Competing Interest Statement

The authors have no competing interests for this work.
} 


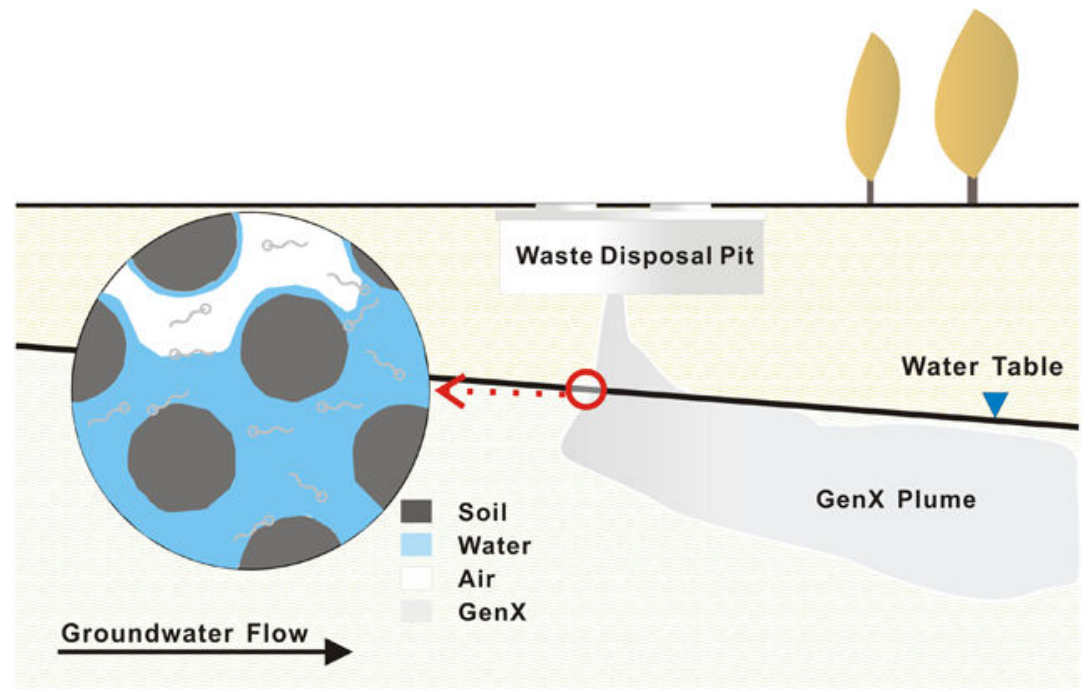

\section{Keywords}

PFAS; air-water interface; adsorption; surface tension

\section{Introduction}

Per- and poly-fluoroalkyl substances (PFAS) are emerging contaminants of critical concern in many industrialized nations due to their widespread occurrence, high persistence, and potential toxicity ${ }^{1-4}$. Because of these concerns, the common longer-chain PFAS perfluorooctanoic acid (PFOA) and perfluorooctanesulfonic acid (PFOS) have been replaced in favor of shorter-chain alternatives such as perfluorohexane sulfonic acid (PFHxS) and perfluoroether carboxylic acids (PFECAs). The shorter-chain PFAS were designed to be less persistent in the environment. However, concern has arisen regarding these shorter-chain PFAS due to their detection in environmental media and potential negative effects on human health ${ }^{4}$.

One shorter-chain replacement is GenX, which has been used commercially since 2009 as a replacement for PFOA. Recent studies have reported the presence of GenX in surface water and groundwater samples collected from different sites ${ }^{5-10}$. For example, GenX has been identified in groundwater near the Chemours facility in North Carolina ${ }^{8}$. GenX was also observed in environmental samples collected near a production plant in The Netherlands ${ }^{9,10}$. According to the recent U.S. EPA draft toxicity assessment, GenX may harm the liver, kidney, immune system, and developing fetus through oral exposure ${ }^{11}$. The North Carolina Department of Health and Human Services (DHHS) provisional health goal for GenX in drinking water is $140 \mathrm{ng} / \mathrm{L}$. The U.S EPA is proposing the draft oral subchronic reference doses (RfDs) of $0.0002 \mathrm{mg} / \mathrm{kg}$-day and chronic RfD of $0.00008 \mathrm{mg} / \mathrm{kg}$-day for GenX ${ }^{12}$. Recent research suggests that GenX may have similar toxicity concerns compared to PFOA $^{13}$. Given its presence in the environment, and the concern over potential toxicity, it is critical to understand the transport and fate behavior of GenX. 
To the best of our knowledge, there have been no prior published investigations of GenX sorption and transport in soil or characterization of adsorption at the air-water interface. The objective of this research is to investigate the retention and transport behavior of GenX in porous media. Surface tensions are measured to characterize surface activity. Miscibledisplacement experiments are conducted under saturated conditions to characterize the magnitude of solid-phase adsorption, while unsaturated-flow experiments are conducted to examine the impact of air-water interfacial adsorption on retention and transport.

\section{Materials and Methods}

\section{Materials}

Two GenX related compounds were used for the experiments: perfluoro (2-methyl-3oxahexanoic) acid (GenX-acid, CAS\# 13252-13-6, Manchester Organics- 97\% purity), and ammonium perfluoro (2-methyl-3-oxahexanoate) $\left(\mathrm{NH}_{4}\right.$-GenX, CAS\# 62037-80-3, Manchester Organics- 95\% purity). Perfluorooctanoic acid (PFOA) and perfluorooctane sulfonic acid (PFOS) were also used for select experiments. Pentafluorobenzoic acid (99\%) was used as the nonreactive tracer (NRT), and was purchased from Strem Chemicals. This compound is not a PFAS and is not a surfactant. It has been widely used as a non-reactive tracer for transport studies employing a diverse range of porous media.

Five porous media were used in the experiments: commercial natural quartz sand, Eustis soil, Vinton soil, Qingdao soil 1 (surface soil, QD1), and Qingdao soil 2 (vadose zone sediment, QD2). The media have similar median grain diameters, but different geochemical properties (Table 1). The primary differences are the organic-carbon and metal-oxide contents. QD1 has the largest organic-carbon content and a low metal-oxide content, whereas Vinton has the largest metal-oxide content and a low organic-carbon content. The other media have a range of organic-carbon contents, but all have relatively low metal-oxide contents. This will provide an opportunity to evaluate the relative influence of those two properties on adsorption.

The miscible-displacement experiments conducted with the sand used $0.01 \mathrm{M} \mathrm{NaCl}$ as the background electrolyte to match prior experiments conducted with other PFAS. A synthetic groundwater (SGW) solution was used as the background electrolyte for the soil experiments. The major cations in this synthetic groundwater (and concentration, $\mathrm{mg} / \mathrm{L}$ ) are $\mathrm{Na}^{+1}(50), \mathrm{Ca}^{+2}(36), \mathrm{Mg}^{+2}(25)$, and major anions are $\mathrm{NO}_{3}{ }^{-1}(6), \mathrm{Cl}^{-1}(60), \mathrm{CO}_{3}{ }^{-2} / \mathrm{HCO}_{3}{ }^{-1}$ (133), and $\mathrm{SO}_{4}^{-2}$ (99). The $\mathrm{pH}$ and ionic strength of the groundwater solution are 7.7 and $0.01 \mathrm{M}$, respectively. Solutions were prepared using distilled, deionized water.

\section{Surface-tension Measurements}

The surface tensions of aqueous GenX solutions (deionized water, $0.01 \mathrm{M} \mathrm{NaCl}$, and $\mathrm{SGW}$ ) were measured using a De Nouy ring tensionmeter (Fisherscientific, Surface Tensiomat 21) following standard methods. The tensiometer was calibrated with a weight of known mass. Each sample was measured three times with the deviation between measurements less than $1 \%$. 
Fluid-fluid interfacial adsorption coefficients can be determined from the surface/interfacial tension function. The surface excess $\Gamma\left(\mathrm{mol} / \mathrm{cm}^{2}\right)$ of a compound at the interface is related to the aqueous-phase concentration $(\mathrm{C})$ using the Gibbs equation:

$$
\Gamma=\frac{-1}{\mathrm{xRT}} \frac{\partial \gamma}{\partial \ln C}
$$

where $\gamma$ is the interfacial tension ( $\mathrm{dyn} / \mathrm{cm}$ or $\mathrm{mN} / \mathrm{m}$ ), $\mathrm{C}$ represents the aqueous phase concentration $\left(\mathrm{mol} / \mathrm{cm}^{3}\right), \mathrm{T}$ is temperature $\left({ }^{\circ} \mathrm{K}\right), \mathrm{R}$ is the universal gas constant (dyne$\mathrm{cm} / \mathrm{mol}^{\circ} \mathrm{K}$ ), and $x$ is a coefficient equal to 1 for systems with nonionic surfactants or ionic surfactants with excess electrolyte in solution, and equal to 2 for systems with ionic surfactants without excess electrolyte (e.g., deionized water). The Gibbs equation has been shown in innumerable studies to provide accurate representation of surfactant adsorption at fluid-fluid interfaces.

The amount adsorbed at the interface as a function of aqueous concentration can be determined as ${ }^{14-17}$ :

$$
K_{i}=\frac{\Gamma}{C}=\frac{-1}{\mathrm{xRTC}} \frac{\partial \gamma}{\partial \ln C}
$$

where $\mathrm{K}_{\mathrm{i}}$ is the interfacial adsorption coefficient $(\mathrm{cm}) . \mathrm{K}_{\mathrm{i}}$ can be determined for any given aqueous concentration by calculating the local slope of surface tension versus $\ln \mathrm{C}$ (i.e., $\partial \gamma /(\partial \ln C)$ in equations 1 and 2) through use of a tangent taken at the concentration of interest $^{15,16}$, and dividing by the relevant $\mathrm{C}$ (equation 2). It is important to note that this approach employs measured surface-tension data and equation 2 to calculate $\Gamma$ and $\mathrm{K}_{\mathrm{i}}$ values directly, without the need to specify an adsorption-isotherm model. A mathematical function is typically used to aid in the determination of slopes. The Szyszkowski equation was applied to the measured surface-tension data sets to support the determination of $\Gamma$ values. Numerous authors have demonstrated that the Szyszkowski equation provides accurate representation of surfactant surface-tension and interfacial-tension data ${ }^{14,17-22}$, including for PFAS $^{23-25}$. One form of the equation is given as ${ }^{14,18}$ :

$$
\gamma=\gamma_{0}\left[1-B \ln \left(1+\frac{C}{A}\right)\right]
$$

where $\gamma_{0}$ is the interfacial tension at [PFAS] $=0$ (e.g., the surface tension of pure water), and $A$ and $B$ are variables related to properties of the specific compound and of the homologous series, respectively. The best-fit functions were used to obtain the slope factors required for equation 2 for all data sets.

\section{Batch Adsorption Experiments}

Batch experiments were conducted to attempt to measure adsorption of GenX. The methods used were identical to those used successfully in our recent study on PFOS adsorption and transport in soil ${ }^{33}$. Four initial concentrations of $10 \mu \mathrm{g} / \mathrm{L}, 100 \mu \mathrm{g} / \mathrm{L}, 1 \mathrm{mg} / \mathrm{L}$, and $10 \mathrm{mg} / \mathrm{L}$ were selected to match the range spanned by the column experiments. It is important to note that the experiments used a solution:soil ratio of 2:1. Inspection of the literature shows that 
the ratios used for PFAS batch experiments are typically 10:1 or greater (often significantly greater). The significantly lower ratio was used in light of the anticipated low magnitudes of adsorption.

The final aqueous concentrations for many of the samples containing soil were within statistical uncertainty of the samples containing no soil (controls). Therefore, the results were indeterminate. This outcome was not unexpected and it illustrates a disadvantage of batch methods for solute-media pairs that exhibit very low sorption. Column experiments offer a more robust approach in these cases due to the much lower solution:soil ratios $(\sim 0.2)$.

\section{Miscible-displacement Column Experiments}

The columns used in this study were constructed of acrylic to minimize interaction with GenX, and were $15 \mathrm{~cm}$ long with inner diameters of $2.6 \mathrm{~cm}$. Porous plates constructed of polypropylene were placed in contact with the porous media on the top and at the bottom of the column to support the media and to help promote uniform distribution of solutions. Precision HPLC pumps were used to provide constant flow of solution. Tubing and samplecollection vials were constructed of high density polyethylene.

The miscible-displacement experiments were conducted with $\mathrm{NH}_{4}$-GenX. The experiments for each medium were conducted with one or more input concentrations $\left(\mathrm{C}_{0}\right)$ of $10 \mu \mathrm{g} / \mathrm{L}, 1$ $\mathrm{mg} / \mathrm{L}$, and $10 \mathrm{mg} / \mathrm{L}$. The focus of this study is on GenX transport behavior in soil and the vadose zone at source zones. Therefore, the $\mathrm{C}_{0}$ concentrations were selected in recognition of the fact that PFAS concentrations in soils at contaminated sites are typically significantly larger than groundwater concentrations, and can range up to $\mathrm{mg} / \mathrm{kg}$ levels ${ }^{26}$. These levels have been reported for some of the primary constituents of AFFF, and few soils data have been reported specifically for GenX. However, recent research has demonstrated the presence of high levels of GenX in soil.

Gebbink and van Leeuwen reviewed published data on GenX concentrations in soil and groundwater reported for multiple sampling sites in the Netherlands ${ }^{27}$. The mean soil concentrations for four studies ranged from $<0.1$ to $132 \mu \mathrm{g} / \mathrm{kg}$, while the mean groundwater concentrations ranged from $<0.02$ to $18 \mu \mathrm{g} / \mathrm{L}$ One study reported concentrations for 6 sites that were located approximately $1 \mathrm{~km}$ or greater from a plant that processes GenXcontaining products. Soil concentrations for the closest site ranged up to a maximum of 1.3 $\mathrm{mg} / \mathrm{kg}$. Concentrations reported for the 6 sites were used herein to calculate soil:groundwater concentration ratios. The ratio for the closest site is 7.3; the geomean for all sites is 7. Soil concentrations were higher than groundwater concentrations for the two other studies for which both types of samples were collected. Hence, soil concentrations are significantly greater than groundwater concentrations for all three studies. In another study, GenX soil concentrations ranging up to $28 \mu \mathrm{g} / \mathrm{kg}$ were reported for a manufacturing plant in $\mathrm{NC}^{28}$. These results demonstrate that GenX concentrations can range into the $10 \mathrm{~s}$ and $100 \mathrm{~s}$ of $\mu \mathrm{g} / \mathrm{kg}$ and higher, in soil. It is particularly noteworthy that a concentration exceeding 1 $\mathrm{mg} / \mathrm{kg}$ was reported for a site $\sim 1 \mathrm{~km}$ from the source.

The columns were packed with air-dried media to obtain uniform bulk densities. The columns were oriented vertically for all experiments. Each column was first saturated with 
water by introducing water at a low flow rate $(\sim 0.05 \mathrm{~mL} / \mathrm{min})$ into the bottom of the column. Saturated-flow experiments were conducted with the nonreactive tracer to characterize the hydrodynamic properties of the packed columns. Experiments were then conducted with GenX to characterize the nature and impact of solid-phase sorption on transport. Additional experiments were conducted with PFOS and PFOA for the sand and Eustis soil to allow a comparison of adsorption among the three PFAS. The conditions for these additional experiments are noted in Table SI1. The experiments were conducted with a flow rate of 1 $\mathrm{mL} / \mathrm{min}$, equivalent to a mean pore-water velocity of $\sim 25 \mathrm{~cm} / \mathrm{hr}$. A step-input approach was used to ensure that effluent concentrations attained $\mathrm{C} / \mathrm{C}_{0}=1$.

For the unsaturated-flow experiments, the top cap was removed to expose the surface to the atmosphere. A thin layer of glass beads was placed on the medium surface to help distribute solution and protect the surface from disruption. Two methods were employed to maintain steady unsaturated flow. For the first method, tubing from the bottom of the column was connected to a vacuum chamber that housed a fraction collector to which the column effluent line was connected. The chamber was connected to a vacuum pump. A HPLC pump was used to provide constant water flow to the exposed top of the column. For the second method, two pumps were connected to the column, one to the top where solution was injected and one to the bottom where solution was withdrawn. Manipulation of the injection flow rate and the magnitude of vacuum or extraction flow rate provides for controlled drainage to a selected water content. Once steady state conditions were achieved, miscibledisplacement experiments were conducted for the nonreactive tracer and GenX. The mass of the column was measured periodically to track changes due to drainage, and to determine the final water content. These methods have been demonstrated in our prior work to produce steady unsaturated flow conditions ${ }^{29-32}$. Note that unsaturated-flow experiments could not be conducted for the QD1 soil due to its high soil-moisture retention capacity.

\section{Analytical Methods}

The PFBA samples were analyzed by ultraviolet-visible (UV-Vis) spectrophotometry (Shimadzu, model 1601) at wavelength of $262 \mathrm{~nm}$. The quantitative detection limit (QDL) for UV-Vis is approximately $1 \mathrm{mg} / \mathrm{L}$. GenX was analyzed by two methods. The first method employed Thermo Ultimate 3000 HPLC (Thermo Fisher Scientific, Breme, Germany) coupled with an AB-Sciex Qtrap 4500 mass spectrometer (AB Sciex Pte. Ltd, Singapore) with an electrospray ionization source. A Waters C18 $2.1 \times 50 \mathrm{~mm}, 1.7 \mu \mathrm{m}$ particle size analytical column was used. Column temperature was maintained at $30^{\circ} \mathrm{C}$. The dual mobile phase was comprised of $2 \mathrm{mM}$ ammonium acetate and 100\% methanol applied in a 30:70 gradient at a flow rate of $0.2 \mathrm{~mL} / \mathrm{min}$ for $5 \mathrm{~min}$. Samples of column effluent were diluted and passed through a $0.45 \mu \mathrm{m}$ pore size membrane before analyzed with the injection volumes of $2 \mu \mathrm{l}$ Retention time was consistently $\sim 1.02 \mathrm{~min}$. The quantifiable detection limit is $\sim 0.1 \mu \mathrm{g} / \mathrm{L}$.

The second method employed the methylene blue active substances (MBAS) assay. This is a standard method used to quantify anionic surfactant concentrations (ASTM D2330-02), and has been used successfully for PFAS analysis under conditions wherein a single PFAS is present $^{32}$. This method was used for most of the experiments with $\mathrm{C}_{0}$ of $10 \mathrm{mg} / \mathrm{L}$. The 
quantifiable detection limit is $\sim 0.4 \mathrm{mg} / \mathrm{L}$. The two methods have been shown to produce consistent results ${ }^{33}$.

\section{Data Analysis}

The retardation factor $(\mathrm{R})$ for aqueous-phase transport of solute undergoing retention by adsorption to solid-water and air-water interfaces is given as ${ }^{29-32,34}$ :

$$
R=1+\frac{K_{d} \rho_{b}}{\theta_{w}}+\frac{K_{i} A_{a w}}{\theta_{w}}
$$

where $K_{d}$ is the solid-phase adsorption coefficient $\left(\mathrm{cm}^{3} / \mathrm{g}\right), K_{i}$ is the air-water interfacial adsorption coefficient $\left(\mathrm{cm}^{3} / \mathrm{cm}^{2}\right), A_{a w}$ is the specific air-water interfacial area $\left(\mathrm{cm}^{2} / \mathrm{cm}^{3}\right), \rho_{b}$ is porous-medium bulk density $\left(\mathrm{g} / \mathrm{cm}^{3}\right), \theta_{W}$ is volumetric water content $(-)$, and $\theta_{\mathrm{w}}+\theta_{\mathrm{a}}=\mathrm{n}$, where $\theta_{a}$ is volumetric air content and $n$ is porosity.

Measured retardation factors were determined for each column experiment by the standard methods of calculating the area above the breakthrough curve and by temporal moment analysis. It has been widely demonstrated theoretically and experimentally that $R$ and $K_{d}$ values determined by these methods are not influenced by the impact of rate-limited or nonlinear adsorption (e.g. ${ }^{35-37}$ ). Column experiments have been demonstrated in numerous studies to produce robust measures of adsorption (e.g., ${ }^{36-38}$ ). In addition, it has been demonstrated that $\mathrm{K}_{\mathrm{d}}$ values measured from column experiments are consistent with those determined with batch experiments, as long as consistent experiment and data-analysis methods are used for both. These points were addressed in a recent study of PFOS adsorption and transport in soil, for which it was demonstrated that column experiments produced representative measures of adsorption and that $K_{d}$ values were consistent between batch and column experiments ${ }^{33}$.

These retardation factors incorporate the contributions of all relevant retention processes influencing transport. For saturated-flow conditions, $K_{d}$ is obtained by solving the reduced form of equation 4 . When adsorption is nonlinear, these values represent the equivalent $K_{d}$ for the respective $\mathrm{C}_{0}$ used in the particular experiment. The fraction of total retention associated with air-water interfacial adsorption is calculated as: $\mathrm{F}_{\mathrm{awia}}=\left(\mathrm{R}-1-\left(\mathrm{K}_{\mathrm{d}} \mathrm{P}_{\mathrm{b}} / \theta_{\mathrm{w}}\right)\right) /$ $(\mathrm{R}-1)=\left(\mathrm{K}_{\mathrm{i}} \mathrm{A}_{\mathrm{aw}} / \theta_{\mathrm{w}}\right) /(\mathrm{R}-1)$. The retention term associated with adsorption at the air-water interface is defined as AWIA $=\mathrm{K}_{\mathrm{i}} \mathrm{A}_{\mathrm{aw}} / \theta_{\mathrm{w}}$.

\section{Mathematical Modeling}

The breakthrough curves for the nonreactive tracer were simulated with a one-dimensional numerical model representing the standard advection-dispersion equation with ideal transport. The dispersivity values were obtained by calibration of the model to the nonreactive tracer breakthrough curves using a nonlinear least-squares optimization program. The breakthrough curves for GenX transport under saturated-flow conditions were simulated with an advection-dispersion model incorporating the standard two-domain approach to represent nonlinear, rate-limited solid-phase adsorption-desorption. The dispersivity obtained from analysis of the nonreactive tracer data is used for these simulations. The retardation factor was measured independently by moment analysis of the 
breakthrough curves. The parameters $\beta$ and $\omega$, representing rate-limited sorption/desorption effects on transport, are obtained by calibration. A Freundlich $\mathrm{N}$ value of 0.89 , based on the results of the column isotherm data, was used for the simulations incorporating nonlinear solid-phase adsorption. Simulations assuming linear adsorption were also conducted for comparison. The relevant equations for these models are presented in recent publications $\mathrm{s}^{33,39}$ and are not repeated herein.

The transport of GenX under unsaturated conditions was simulated with a model that accounts explicitly for rate-limited solid-phase adsorption and rate-limited air-water interfacial adsorption ${ }^{39}$. This model was used in a predictive mode, with values for all input parameters obtained independently (no calibration to the unsaturated-flow BTC data). The dispersivity was obtained from the unsaturated-flow nonreactive tracer data. The retardation factor was obtained by moment analysis of the breakthrough curves. The $\mathrm{K}_{\mathrm{d}}, \mathrm{k}_{2}$, and $\mathrm{F}$ values were obtained from the saturated-flow GenX experiments. The rate coefficient for Genx adsorption at the air-water interface is not known. Therefore, three sets of simulations were conducted, varying the magnitude of this parameter. For the first set, AWIA was treated as instantaneous $\left(a_{n w}=50, \omega=100\right)$. AWIA was treated as rate limited for the second set of simulations, with a rate coefficient selected to produce a moderate degree of nonequilibrium $\left(a_{n w}=2, \omega=5\right)$. The third set of simulations employed a rate coefficient that produced a greater degree of nonequilibrium $\left(a_{n w}=0.2, \omega=0.5\right)$.

The Damkohler Number, $\omega$ is a nondimensional mass-transfer rate coefficient representing the ratio of residence time and characteristic time of mass transfer. It serves as an index characterizing the degree to which a mass-transfer process is rate limited with respect to advective transport. The $\omega$ for rate-limited air-water interfacial adsorption is defined as $\omega=$ $a_{n w} L / q$, where $a_{n w}$ is the first-order mass transfer coefficient for air-water interfacial adsorption $(1 / T)$, $q$ is specific discharge $(\mathrm{L} / \mathrm{T})$, and $\mathrm{L}$ is system length $(\mathrm{L})^{39}$. An $\omega$ of 100 represents the effective upper limiting case wherein mass transfer is sufficiently rapid compared to residence time that it can be treated as effectively instantaneous. Conversely, a value of 0.01 represents the effective lower limiting case for which mass transfer is extremely rate limited with respect to advective transport.

\section{Results and Discussion}

\section{Surface Tension Data}

Surface tensions for GenX-acid and $\mathrm{NH}_{4}$-GenX in the three solutions are presented in Figure 1. The addition of salts increased surface activities, consistent with the general impact of ionic strength on surface tensions of surfactants. The largest surface activities for both compounds are measured for the SGW solution. This is to be expected given that this solution contains both monovalent and divalent ions, and is consistent with prior results ${ }^{32,40}$. The impact of solution composition is greater for $\mathrm{NH}_{4}$-GenX than for the acid form because the latter is less readily ionized. Thus, GenX-acid is less affected by the addition of background electrolyte. The Szyszkowski equation is observed to produce good fits to the measured data. 
Adsorption of surfactants at the air-water interface is nonlinear as a function of solution concentration. $\mathrm{K}_{\mathrm{i}}$ values change by approximately two orders of magnitude over the concentration range, with larger values at lower concentrations (Figure SI1). However, the values exhibit minimal change for concentrations less than $\sim 10 \mathrm{mg} / \mathrm{L}$. It is of interest to note that the concentration at which the $\mathrm{K}_{\mathrm{i}}$ values asymptotically approach their maximum is higher than that observed for PFOA, which is in the range of $<1 \mathrm{mg} / \mathrm{L}^{31}$. This disparity is associated with the differences in surface activities of the two, with PFOA exhibiting greater activity compared to GenX.

Maximum $\mathrm{K}_{\mathrm{i}}$ values for GenX-acid determined from the surface-tension data are 0.00023 $\mathrm{cm}$ (DIW), $0.00043 \mathrm{~cm}(\mathrm{NaCl})$, and $0.0006 \mathrm{~cm}(\mathrm{SGW})$. The values for $\mathrm{NH}_{4}-\mathrm{GenX}$ are $0.00014 \mathrm{~cm}$ (DIW), $0.001 \mathrm{~cm}(\mathrm{NaCl})$, and $0.002 \mathrm{~cm}(\mathrm{SGW})$. The $\mathrm{K}_{\mathrm{i}}$ value for GenX-acid is approximately 3 times larger in SGW versus DI water. Conversely, the $\mathrm{K}_{\mathrm{i}}$ value for $\mathrm{NH}_{4}$ GenX is $\sim 14$ times larger. These results illustrate the significant impact of ionic strength on air-water interfacial adsorption of GenX. Concentrations of GenX reported in soil and groundwater are typically less than $1 \mathrm{ppm}$. Thus, the maximum $\mathrm{K}_{\mathrm{i}}$ values reported above are anticipated to be representative for most environmental systems. These results also indicate that employing $\mathrm{C}_{0}$ values of $10 \mathrm{mg} / \mathrm{L}$ and lower for the miscible-displacement experiments essentially captures the maximum impact of air-water interfacial adsorption.

Brusseau $^{25}$ conducted a quantitative-structure/property-relationship (QSPR) analysis of fluid-fluid interfacial adsorption coefficients for 42 individual PFAS. The PFAS evaluated comprised homologous series of perfluorocarboxylates and perfluorosulfonates, branched perfluoroalkyls, polyfluoroalkyls, alcohol PFAS, and nonionic PFAS. The $\mathrm{K}_{\mathrm{i}}$ values varied across eight orders of magnitude, and were demonstrated to be a function of molecular structure. A QSPR model employing molar volume $\left(\mathrm{V}_{\mathrm{m}}\right)$ as a descriptor provided robust predictions of $\log \mathrm{K}_{\mathrm{i}}$ values for air-water and OIL-water interfacial adsorption coefficients. A follow-up study presented a revised QSPR model that incorporated the impact of ionic strength among other factors ${ }^{40}$. The $\log K_{i}$ values predicted for GenX with the QSPR models are similar to the measured maximum values. For example, the measured maximum $\log \mathrm{K}_{\mathrm{i}}$ for DI water is -3.9 versus a predicted value of -4.1 obtained from the original model (which employed data measured in DI water). Similarly, the measured maximum $\log \mathrm{K}_{\mathrm{i}}$ for $0.01 \mathrm{M} \mathrm{NaCl}$ is -3.1 versus a predicted value of -3.4 obtained from the revised model that accounts for the presence of salts. These results indicate that the air-water interfacial adsorption of GenX is consistent with that of other PFAS.

\section{GenX Transport for Saturated Conditions}

The breakthrough curves (BTCs) for transport of the nonreactive tracer were sharp and symmetrical, with retardation factors of 1 , for all three porous media (Figure 2). This indicates ideal hydrodynamic conditions for transport in the packed columns. The BTCs for unsaturated-flow conditions exhibited slightly greater spreading compared to saturated conditions for the sand and Vinton media. The solute transport model incorporating only advection and dispersion provides good simulations of the measured BTCs (Figure 2). Overall, the results obtained for the nonreactive tracer tests indicate that the columns were 
well-packed and that water flow was uniform, with no significant preferential flow or presence of no-flow domains for saturated or unsaturated conditions.

The BTCs for GenX transport in the media under saturated conditions exhibit small degrees of retardation (Figure 3), with retardation factors ranging from 1.03 to 1.4 (Table 2). A significantly larger $\mathrm{R}$ of 2.2 was measured for transport in the Vinton soil. These translate to $\mathrm{K}_{\mathrm{d}}$ values of $0.02,0.03,0.04,0.08$ and $0.32 \mathrm{~cm}^{3} / \mathrm{g}$ for the QD2, sand, QD1, Eustis, and Vinton media, respectively. The recoveries are greater than $93 \%$ for all experiments.

Recognizing the potential constraints associated with application of the $\mathrm{K}_{\mathrm{oc}}$ concept to PFAS, $\log \mathrm{K}_{\mathrm{oc}}$ values were determined for discussion purposes using the $\mathrm{f}_{\mathrm{oc}}$ values reported in Table 1. They are 1.48, 1.86, 0.24, 1.34, and 2.55 for QD2, sand, QD1, Eustis, and Vinton, respectively. For comparison, a value of 1.8 is predicted using a $\log \mathrm{K}_{\mathrm{oc}}-\mathrm{V}_{\mathrm{m}}$ regression reported for other PFAS ${ }^{41}$. Two of the values are significant outliers from this predicted value. The value for the QD1 soil, which has the largest organic-carbon content, is very small. It is observed that the $\mathrm{K}_{\mathrm{d}}$ and $\log \mathrm{K}_{\mathrm{oc}}$ values for Eustis soil, which has an appreciable but 6-times lower organic-carbon content (but a similar metal-oxide content), are larger than for QD1. Geochemical analysis of the Eustis organic carbon reported approximately 37\% hard carbon (kerogen and black carbon) and 63\% soft carbon (humic/fulvic acids, lipids) ${ }^{42}$. The QD1 soil was collected from an agricultural field. Hence, it may be speculated that the organic carbon may be relatively young and thus comprised primarily of soft carbon constituents.

Conversely, the $\log \mathrm{K}_{\mathrm{oc}}$ value for the Vinton soil is significantly larger than for all of the other media, and is also larger than the regression value. It is notable that while Vinton soil has a very low organic-carbon content, it has by far the largest metal-oxide content. The greater proportion of oxides likely supports adsorption via additional mechanisms beyond hydrophobic interaction, such as electrostatic interactions and the formation of complexes by ligand exchange with metal oxides, as has been observed in prior studies for PFAS and hydrocarbon surfactants ${ }^{43-48}$. This additional adsorption is lumped into the $\log \mathrm{K}_{\mathrm{oc}}$ value. A similar observation of greater adsorption by Vinton compared to Eustis soil was reported for PFOS $^{33}$.

The results of new experiments and those previously reported for transport of PFOA and PFOS in the sand and Eustis soil are presented in Table SI1, with a comparison to the relevant GenX results. $K_{d}$ values of 0.08 and $0.17 \mathrm{~cm}^{3} / \mathrm{g}$ for $\mathrm{C}_{0}=1 \mathrm{mg} / \mathrm{L}$ were reported for PFOA and PFOS, respectively, for transport in the sand, compared to a value of $0.02 \mathrm{~cm}^{3} / \mathrm{g}$ for GenX. Similar differences in $K_{d}$ values for the three are observed for $C_{0}=10 \mathrm{mg} / \mathrm{L}$ experiments (Table SI1). $K_{d}$ values of 0.1 and $0.4 \mathrm{~cm}^{3} / \mathrm{g}$ were determined for PFOA and PFOS, respectively, for transport in Eustis soil, compared to a value of $0.08 \mathrm{~cm}^{3} / \mathrm{g}$ for GenX. The differences are consistent with the sand results. The $\mathrm{K}_{\mathrm{d}}$ values for GenX are consistently the smallest, with those for PFOA intermediate and those for PFOS the largest. The $\log \mathrm{K}_{\mathrm{oc}}$ values also exhibit this trend. These results are consistent with that expected based on the different chain lengths and molar volumes of the three. 
The BTCs for GenX transport under saturated conditions are asymmetrical, exhibiting asymptotic approaches to relative concentrations of 1 and 0 . The observation of tailing for both arrival and elution waves, in conjunction with the ideal transport observed for the nonreactive tracer, suggests that GenX transport is influenced by rate-limited sorptiondesorption. Inspection of Table 2 shows that the retardation factors are slightly larger for the lowest $\mathrm{C}_{0}$ experiments, indicating an influence of nonlinear adsorption. The $\mathrm{K}_{\mathrm{d}}$ values are $0.014,0.016$, and $0.029 \mathrm{~cm}^{3} / \mathrm{g}$ for the $\mathrm{C}_{0}=10,1$, and $0.01 \mathrm{mg} / \mathrm{L}$ experiments, respectively, for the sand. The $\mathrm{K}_{\mathrm{d}}$ data can be used to generate an adsorption isotherm (Figure SI2). It is observed that the Freundlich equation provides a good fit to the measured data. The Freundlich $\mathrm{N}$ term is 0.89 , indicating a small degree of nonlinearity.

The relative influence of rate-limited and nonlinear adsorption on GenX transport can be assessed through mathematical modeling. The simulations produced with the transport model incorporating two-domain rate-limited adsorption-desorption provide reasonable matches to the measured GenX BTCs (Figure SI3-5). Simulations produced with and without including nonlinear adsorption are very similar (Figure SI5). It is noted that the simulation testing the impact of nonlinear adsorption was conducted using an $\mathrm{N}$ value of 0.6 , which is likely smaller than actual values. Even with this smaller value, nonlinear adsorption has minimal impact on the BTC. This indicates that rate-limited adsorption is of greater significance compared to nonlinear adsorption for GenX transport in these media. Similar results were observed for the transport of PFOS in soil ${ }^{33}$. The parameters obtained from the modeling are presented in Table SI2. The first-order rate coefficient ranges from 0.3 to $\sim 1 \mathrm{~h}$

${ }^{-1}$. These values are consistent with those $\left(0.1\right.$ to $\left.5 \mathrm{~h}^{-1}\right)$ reported for a batch kinetic study of PFOS adsorption by several soils ${ }^{49}$ and to those reported $\left(0.5\right.$ to $\left.3 \mathrm{~h}^{-1}\right)$ for miscibledisplacement experiments for PFOS transport in two soils ${ }^{33}$. Interestingly, the $\mathrm{F}$ values are essentially 0 for the sand and Eustis soil, but is significantly greater than 0 for Vinton soil.

\section{GenX Transport for Unsaturated Conditions}

The BTCs for GenX transport under unsaturated conditions exhibit greater retardation compared to saturated conditions (see Figure 3 for an illustration), with attendant larger retardation factors (Table 2). These results reflect the impact of the additional retention process associated with adsorption at the air-water interface that occurs under unsaturated conditions. This is consistent with prior results reported for PFOA and PFOS transport in unsaturated media ${ }^{31,32}$.

Air-water interfacial adsorption (AWIA) contributes 58-80\% of the overall retention of GenX in the sand, QD2 soil, and Eustis soil. Conversely, the contribution of air-water interfacial adsorption is much smaller for Vinton soil (24\%). The much lower contribution of air-water interfacial adsorption for Vinton is due to the greater significance of solid-phase adsorption for this medium. These magnitudes of relative contributions are in the range of those determined for PFOA and PFOS in prior studies ${ }^{31,32}$. The AWIA values are reported in Table 2. For a given compound and concentration (i.e., same $\mathrm{K}_{\mathrm{i}}$ ), the magnitudes are a function of the water content and air-water interfacial area. For example, it is observed that the AWIA value for Vinton is larger than those for the sand for similar water saturations. This is due to Vinton having a larger air-water interfacial area ${ }^{29,50}$. 
Air-water interfacial areas measured in prior research for the sand are in the range of 90-100 $\mathrm{cm}^{-1}$ for the range of water saturations of the GenX experiments ${ }^{50}$. With known values for $\mathrm{A}_{\mathrm{aw}}$ and $\mathrm{K}_{\mathrm{d}}$ (the latter determined from the saturated-flow experiments), the measured retardation factors from the unsaturated-flow experiments can be used with Equation 4 to calculate $K_{i}$ values for air-water interfacial adsorption. A mean value of $0.001 \mathrm{~cm}^{-1}$ is calculated for the lowest input-concentration experiments. It is noteworthy that this matches the maximum $\mathrm{K}_{\mathrm{i}}$ value of $0.001 \mathrm{~cm}^{-1}$ determined from the measured surface-tension data.

The two-domain advection-dispersion transport model used for saturated-flow conditions can produce adequate simulations of GenX transport under unsaturated conditions (data not shown). However, such a simulation produces lumped kinetics parameters given that the two retention processes are combined. A more appropriate modelling approach would be to employ a distributed-process model that accounts separately for both rate-limited solidphase adsorption and rate-limited AWIA ${ }^{39}$. Such a model was applied to the GenX data, as shown in Figure 4.

Three sets of independently predicted simulations were conducted to investigate the influence of rate-limited AWIA. For the first set, AWIA was treated as instantaneous $(\omega=$ 100). Inspection of the figures show that this simulation provides good matches to the measured breakthrough curves. AWIA was treated as rate limited for the second set of simulations, and a rate coefficient was selected to produce a moderate magnitude of nonequilibrium $(\omega=5)$. The simulations produced for this case are very similar to those produced for the case of instantaneous AWIA. This is illustrated in Figure 4B for Eustis soil, the medium for which the differences were the greatest. The third set of simulations employed a rate coefficient that produced a greater degree of nonequilibrium $(\omega=0.5)$. The simulated curves deviate from the measured data (Figure 4B). The results of these simulations indicate that AWIA of GenX can be treated as effectively instantaneous under the conditions of the experiments. This is consistent with the results reported for PFOA and PFOS $^{39}$.

\section{Implications}

The results of these experiments show that the magnitudes of solid-phase adsorption of GenX are relatively small. Air-water interfacial adsorption provided significant additional retention under unsaturated conditions. The additional retention resulted in greater retardation of GenX. Both solid-phase and air-water interfacial adsorption of GenX were of smaller magnitudes compared to PFOA and PFOS, consistent with their respective molecular structures. Overall, GenX retardation was relatively low for a range of soil types, indicating that GenX has appreciable migration potential in soil and the vadose zone and therefore significant potential for impacting groundwater. These results suggest that it is critical to characterize concentration-depth profiles of GenX at contaminated sites to determine the extent of migration.

\section{Supplementary Material}

Refer to Web version on PubMed Central for supplementary material. 


\section{Acknowledgements}

This work was supported by the National Natural Science Foundation of China (NSFC) (41907161), the NIEHS Superfund Research Program (grant\# P42 ES04940), and the State Key Program of National Natural Science Foundation of China (41731280). We thank Sarah van Glubt and Dandan Huang for their assistance with the PFOS and PFOA experiments. We thank Lingyan Ma in the Center of Instrument of College of Environmental Science and Engineering, Ocean University of China, for her assistance with LCMS analyses. We thank the reviewers for their comments.

\section{References}

(1). Ahrens L Polyfluoroalkyl compounds in the aquatic environment: a review of their occurrence and fate. J. Environ. Monit 2011, 13, 20-31. [PubMed: 21031178]

(2). EPA, $2019 \mathrm{EPA}^{\mathrm{TM}} \mathrm{S}$ per- and polyfluoroalkyl substances (PFAS) action plan. U.S. Environmental Protection Agency, Washington, DC.

(3). NGWA, 2017 Groundwater and PFAS: State of Knowledge and Practice. National Ground Water Association, Westerville, $\mathrm{OH}$.

(4). Birnbaum LS; Grandjean P Alternatives to PFASs: Perspectives on the science. Environ. Health Perspect 2015, 123 (5), A104-105. [PubMed: 25932670]

(5). Pan Y; Zhang H; Cui Q; Sheng N; Yeung LWY; Sun Y; Guo Y; Dai J Worldwide distribution of novel perfluoroether carboxylic and sulfonic acids in surface water. Environ. Sci. Technol 2018, 52, 7621-7629. [PubMed: 29749740]

(6). Heydebreck F; Tang J; Xie Z; Ebinghaus R Alternative and legacy perfluoroalkyl substances: Differences between European and Chinese river/estuary systems. Environ. Sci. Technol 2015, 49, 8386-8395. [PubMed: 26106903]

(7). Strynar M; Dagnino S; McMahen R; Liang S; Lindstrom A; Andersen E; McMillan L; Thurman M; Ferrer I; Ball C Identification of novel perfluoroalkyl ether carboxylic acids (PFECAs) and sulfonic acids (PFESAs) in natural waters using accurate mass time-of-flight mass spectrometry (TOFMS). Environ. Sci. Technol 2015, 49 (19), 11622-11630. [PubMed: 26392038]

(8). Sun M; Arevalo E; Strynar M; Lindstrom A; Richardson M; Kearns B; Pickett A; Smith C; Knappe D RU. Legacy and emerging perfluoroalkyl substances are important drinking water contaminants in the Cape Fear River Watershed of North Carolina. Environ. Sci. Technol. Lett 2016, 3, 415-419.

(9). Gebbink WA; Van Asseldonk L; Van Leeuwen SP Presence of emerging per-and polyfluoroalkyl substances (PFASs) in river and drinking water near a fluorochemical production plant in the Netherlands. Environ. Sci. Technol 2017, 51, 11057-11065. [PubMed: 28853567]

(10). Brandsma SH; Koekkoek JC; van Velzen MJM; de Boer J The PFOA substitute GenX detected in the environment near a fluoropolymer manufacturing plant in the Netherlands. Chemosphere 2019, 220, 493-500. [PubMed: 30594801]

(11). EPA, 2018 Fact sheet: Draft toxicity assessments for GenX chemicals and PFBS. U.S. Environmental Protection Agency, Washington, DC.

(12). NC HDDS, 2017 NC DHHS preliminary risk assessment FAQ for GenX (Perfluoro2Propoxypropanoic Acid). The North Carolina Department of Health and Human Services, Raleigh, North Carolina.

(13). Gomis MI; Vestergren R; Borg D; Cousins IT Comparing the toxic potency in vivo of long-chain perfluoroalkyl acids and fluorinated alternatives. Environ. Int 2018, 113, 1-9. [PubMed: 29421396]

(14). Barnes G; Gentle I. Interfacial Science: An Introduction. Oxford University Press, New York, NY, 2005.

(15). Hiemenz PC Principles of Colloid and Surface Chemistry, second ed. Marcel Dekker, Inc, New York, 1986.

(16). Fridrikhsberg DA A Course on Colloid Chemistry. Mir Publishers, Moscow, 1986.

(17). Berg JC An Introduction to Interfaces \& Colloids: The Bridge to Nanoscience. World Scientific Publishing Co, Singapore, 2010. 
(18). Adamson AW; Gast AP; Wiley J Physical Chemistry of Surfaces, fourth ed. J. Wiley, New York, 1982.

(19). Fainerman VB; Möbius D; Miller R Surfactants: Chemistry, Interfacial Properties, Applications. Elsevier, Amsterdam, The Netherlands, 2001.

(20). Schick MJ Nonionic Surfactants Physical Chemistry. Marcel Dekker, Inc, New York, 1987.

(21). Zhong H; Ouni A. El; Lin D; Wang B; Brusseau ML The two-phase flow IPTT method for measurement of nonwetting-wetting liquid interfacial areas at higher nonwetting saturations in natural porous media. Water Resour. Res 2016, 52, 5506-5515. [PubMed: 28959079]

(22). Rosen MJ; Kunjappu JT Surfactants and Interfacial Phenomena. John Wiley and Sons, Hoboken, New Jersey, 2012.

(23). Vecitis CD; Park H; Cheng J; Mader BT; Hoffmann MR Enhancement of perfluorooctanoate and perfluorooctanesulfonate activity at acoustic cavitation bubble interfaces. J. Phys. Chem 2008, $112,16850-16857$.

(24). Lunkenheimer K; Prescher D; Hirte R; Geggel K Adsorption properties of surface chemically pure sodium perfluoro-n-alkanoates at the air/water interface: counterion effects within homologous series of 1: 1 ionic surfactants. Langmuir 2014, 31, 970-981.

(25). Brusseau ML The influence of molecular structure on the adsorption of PFAS to fluid-fluid interfaces: Using QSPR to predict interfacial adsorption coefficients. Water Res. 2019, 152, 148 158. [PubMed: 30665161]

(26). Brusseau ML; Anderson RH; Guo B PFAS concentrations in soils: Background levels versus contaminated sites. Sci. Total Environ 2020, 740, article 140017.

(27). Gebbink WA and van Leeuwen SPJ Environmental contamination and human exposure to PFASs near a fluorochemical production plant: Review of historic and current PFOA and GenX contamination in the Netherlands. Environ. Int 2020, 137, 105583. [PubMed: 32106048]

(28). Geosyntec. On and Offsite Assessment: Chemours Fayetteville Works. Project Number TR0795 9 30, 2019.

(29). Brusseau ML; El Ouni A; Araujo JB; Zhong H Novel methods for measuring air-water interfacial area in unsaturated porous media. Chemosphere 2015, 127, 208-213. [PubMed: 25732632]

(30). Brusseau ML; Peng S; Schnaar G; Murao A Measuring air-water interfacial areas with X-ray microtomography and interfacial partitioning tracer tests. Environ. Sci. Technol 2007, 41, 19561961. [PubMed: 17410790]

(31). Lyu Y; Brusseau ML; Chen W; Yan N; Fu X; Lin X Adsorption of PFOA at the air-water interface during transport in unsaturated porous media. Environ. Sci. Technol 2018, 52, 77457753. [PubMed: 29944343]

(32). Brusseau ML; Yan N; Van Glubt S; Wang Y; Chen W; Lyu Y; Dungan B; Carroll KC; Holguin FO Comprehensive retention model for PFAS transport in subsurface systems. Water Res. 2019, 148, 41-50. [PubMed: 30343197]

(33). Brusseau ML; Khan N; Wang Y; Yan N; Van Glubt S; Carroll KC Nonideal transport and extended elution tailing of PFOS in soil. Environ. Sci. Technol 2019, 53, 10654-10664. [PubMed: 31464435]

(34). Brusseau ML Assessing the potential contributions of additional retention processes to PFAS retardation in the subsurface. Sci. Total Environ 2018, 613-614, 176-185.

(35). Valocchi AJ Validity of the local equilibrium assumption for modeling sorbing solute transport through homogeneous soil. Water Resour. Res 1985, 21, 808-820.

(36). Nkedi-Kizza P; Rao PSC; Hornsby AG Influence of organic cosolvents on leaching of hydrophobic organic chemicals through soils. Environ. Sci. Technol 1987, 21, 1107-1111.

(37). Brusseau ML; Rao PSC Sorption nonideality during organic contaminant transport in porous media. CRC Crit. Rev. Environ. Control 1989, 19, 33-99.

(38). Metzelder F; Funck M; Schmidt TC Sorption of heterocyclic organic compounds to multiwalled carbon nanotubes. Environ. Sci. Technol 2018, 52, 628-637. [PubMed: 29257678]

(39). Brusseau ML Simulating PFAS transport influenced by rate-limited multi-process retention. Water Res. 2020, 168, article 115179. 
(40). Brusseau ML; Van Glubt S The influence of surfactant and solution composition on PFAS adsorption at fluid-fluid interfaces. Water Res. 2019, 161, 17-26. [PubMed: 31174056]

(41). Brusseau ML Estimating the relative magnitudes of adsorption to solidwater and air/oil-water interfaces for per- and poly-fluoroalkyl substances. Environ. Pollut 2019, 254, article 113102.

(42). Russo A; Johnson GR; Schnaar G; Brusseau ML Nonideal transport of contaminants in heterogeneous porous media: 8 . Characterizing and modeling asymptotic contaminant-elution tailing for several soils and aquifer sediments. Chemosphere 2010, 81, 366-371. [PubMed: 20692012]

(43). Gao X; Chorover J Adsorption of perfluorooctanoic acid and perfluorooctanesulfonic acid to iron oxide surfaces as studied by flow-through ATR-FTIR spectroscopy. Environ. Chem 2012, 9, 148.

(44). Ochoa-Loza FJ; Noordman WH; Jannsen DB; Brusseau ML; Maier RM Effect of clays, metal oxides, and organic matter on rhamnolipid biosurfactant sorption by soil. Chemosphere 2007, 66, 1634-1642. [PubMed: 16965801]

(45). Zhang R; Yan W; Jing C Experimental and molecular dynamic simulation study of perfluorooctane sulfonate adsorption on soil and sediment components. J. Environ. Sci 2015, 29, 131-138.

(46). Zhang R; Yan W; Jing C Mechanistic study of PFOS adsorption on kaolinite and montmorillonite. Colloids Surfaces A Physicochem. Eng. Asp 2014, 462, 252-258.

(47). Zhao L; Bian J; Zhang Y; Zhu L; Liu Z Comparison of the sorption behaviors and mechanisms of perfluorosulfonates and perfluorocarboxylic acids on three kinds of clay minerals. Chemosphere 2014, 114, 51-58. [PubMed: 25113183]

(48). Westall JC; Chen H; Zhang W; Brownawell BJ Sorption of linear alkylbenzenesulfonates on sediment materials. Environ. Sci. Technol 1999, 18, 3110-3118.

(49). Wei C; Song X; Wang Q; Hu Z Sorption kinetics, isotherms and mechanisms of PFOS on soils with different physicochemical properties. Ecotoxicol. Environ. Saf 2017, 142, 40-50. [PubMed: 28384502]

(50). Araujo JB; Mainhagu J; Brusseau ML Measuring air-water interfacial area for soils using the mass balance surfactant-tracer method. Chemosphere 2015, 134, 199-202. [PubMed: 25950136] 

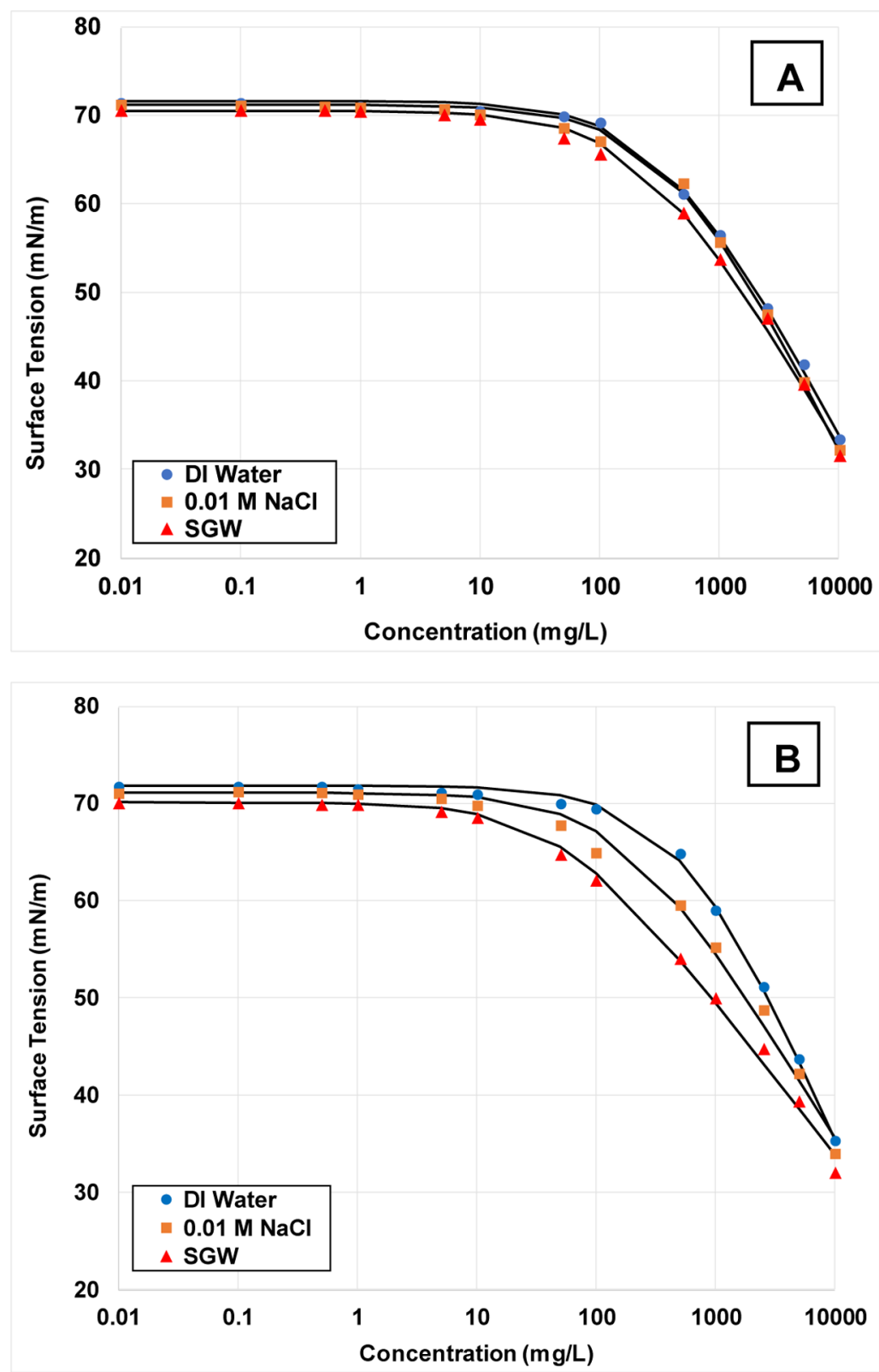

Figure 1.

Surface tensions as a function of GenX concentration in deionized (DI) water, $0.01 \mathrm{M} \mathrm{NaCl}$, and synthetic groundwater (SGW). The solid lines represent the fits of the Szyszkowski equation. (A) GenX acid, (B) $\mathrm{NH}_{4}$ GenX. 

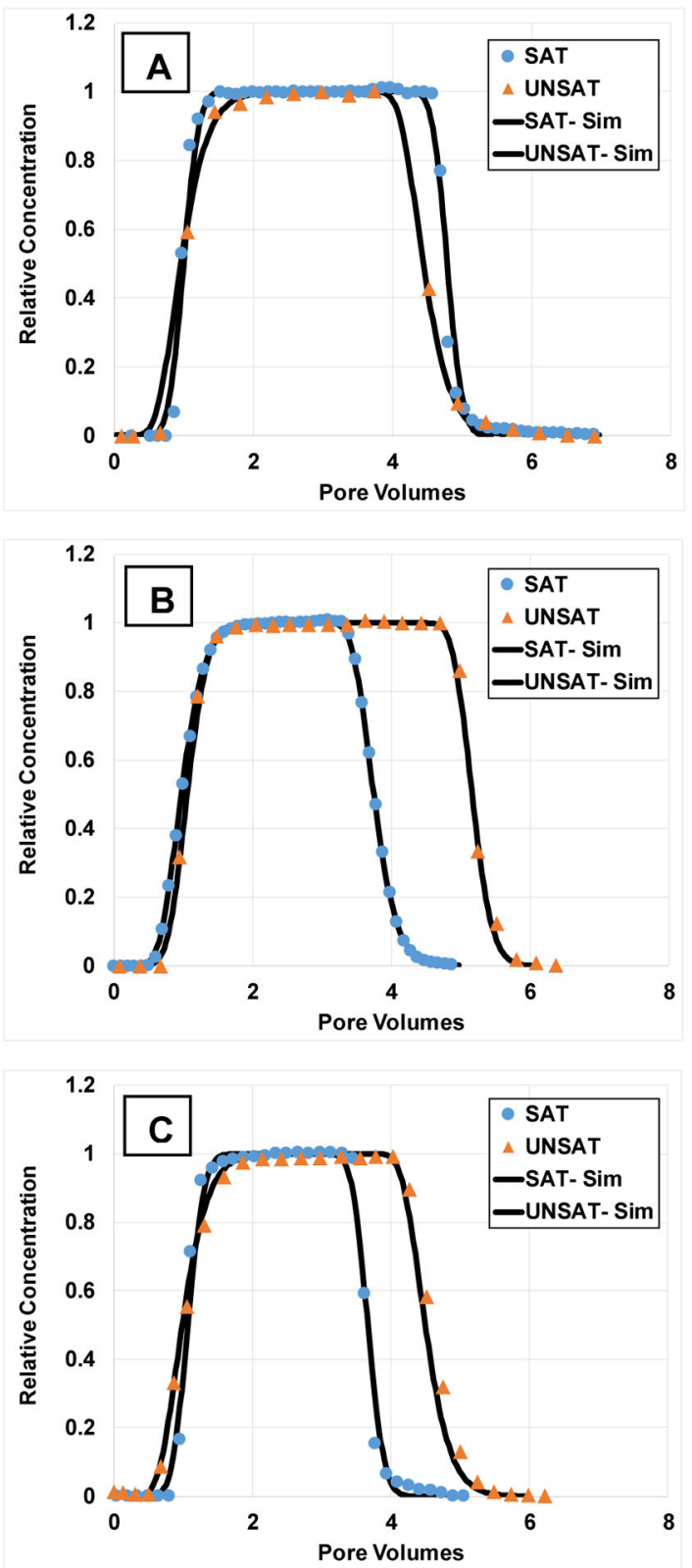

Figure 2.

Breakthrough curves for transport of the nonreactive tracer under saturated (SAT) and unsaturated (UNSAT) conditions. The solid lines represent simulations produced with a 1-D advection-dispersion transport model: (A) sand, (B) Eustis soil, (C) Vinton soil. The pulse size varied for each experiment. 

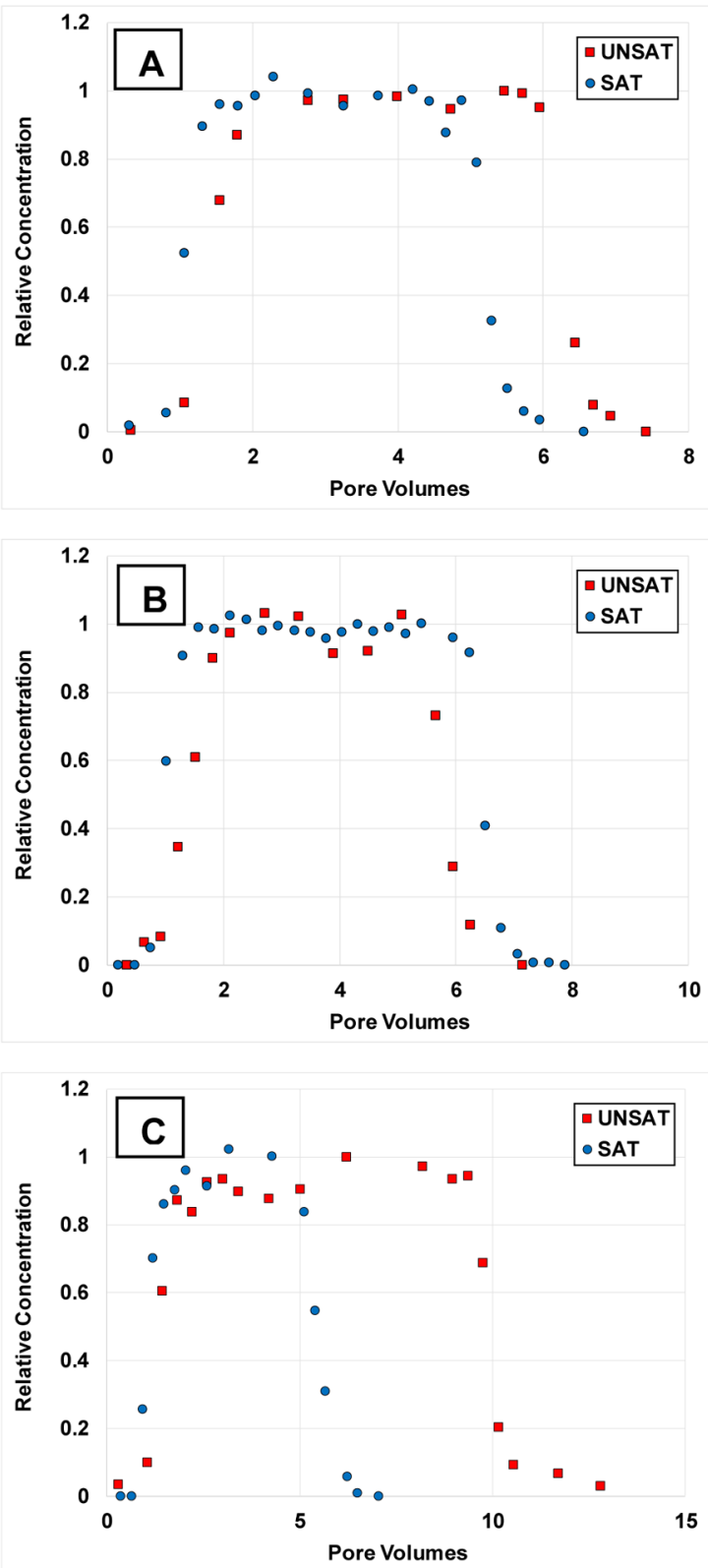

Figure 3.

Breakthrough curves for transport of GenX in the sand under saturated (SAT) and unsaturated (UNSAT) conditions: (A) $\mathrm{C}_{0}=10 \mu \mathrm{g} / \mathrm{mL}$ (B) $\mathrm{C}_{0}=1 \mathrm{mg} / \mathrm{L}$, (C) $\mathrm{C}_{0}=10 \mathrm{mg} / \mathrm{L}$. The pulse size varied for each experiment. 

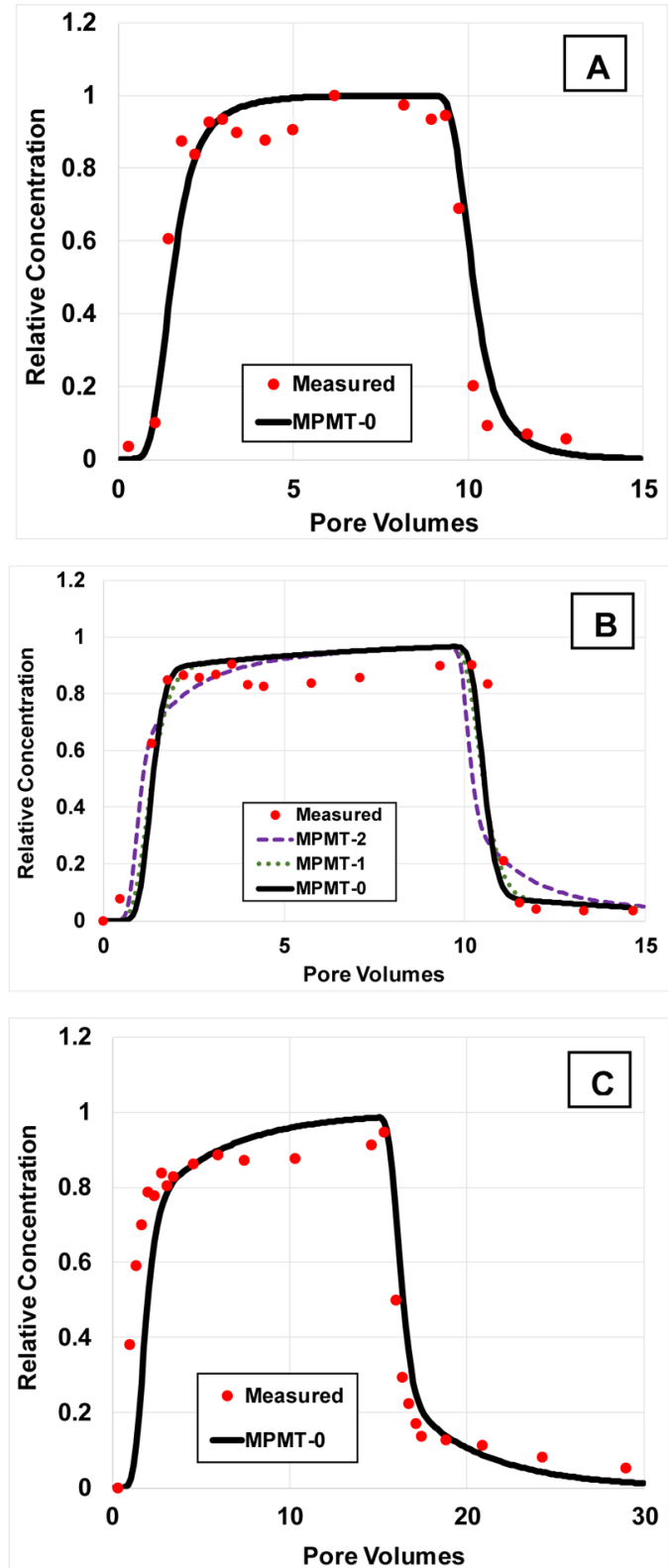

Figure 4.

Breakthrough curves for transport of GenX under unsaturated conditions: (A) sand, (B) Eustis soil, (C) Vinton soil. The simulations represent independent predictions. All cases incorporate rate-limited solid-phase adsorption. Case MPMT-0 treats air-water interfacial adsorption as instantaneous $(\omega=100)$. Cases MPMT- 1 and MPMT-2 treat air-water interfacial adsorption as rate limited ( $\omega=5$ and 0.5 , respectively). 
Table 1.

Properties of porous media.

\begin{tabular}{|c|c|c|c|c|c|c|}
\hline Porous Medium & Sand (\%) & Silt (\%) & Clay (\%) & Median grain diameter d $\mathbf{5 0}_{\mathbf{0}}(\mathbf{m m})$ & TOC $_{(\boldsymbol{\%})}$ & $\mathbf{F e}_{\mathbf{M n}}{ }^{\boldsymbol{b}} \mathbf{\mu g} \mathbf{g}$ \\
\hline QD1 & 73 & 17 & 10 & 0.22 & 2.3 & 328 \\
\hline Eustis & 96 & 1 & 3 & 0.27 & 0.38 & 333 \\
\hline Vinton & 97 & 1.8 & 1.2 & 0.26 & 0.09 & 1,830 \\
\hline QD2 & 90 & 5 & 5 & 0.24 & 0.05 & 365 \\
\hline Sand & 100 & 0 & 0 & 0.35 & 0.04 & 227 \\
\hline
\end{tabular}

total organic carbon

${ }^{b}$ Combined Fe and Mn oxide content 
Table 2.

Results for GenX transport experiments.

\begin{tabular}{|c|c|c|c|c|c|c|}
\hline Experiment & Input Concentration (mg/L) & Water Saturation & Recovery (\%) & Measured R & $\mathbf{F}_{\text {awia }}{ }^{a}$ & AWIA $^{b}$ \\
\hline \multicolumn{7}{|l|}{ Sand } \\
\hline 1 & 0.01 & 1 & 96 & 1.08 & - & - \\
\hline 2 & 0.01 & 1 & 95 & 1.10 & - & - \\
\hline 3 & 1 & 1 & 93 & 1.04 & - & - \\
\hline 4 & 1 & 1 & 95 & 1.06 & - & - \\
\hline 5 & 10 & 1 & 101 & 1.05 & - & - \\
\hline 6 & 10 & 1 & 95 & 1.06 & - & - \\
\hline 7 & 0.01 & 0.65 & 97 & 1.47 & 0.69 & 0.33 \\
\hline 8 & 0.01 & 0.59 & 98 & 1.48 & 0.67 & 0.32 \\
\hline 9 & 1 & 0.63 & 97 & 1.41 & 0.80 & 0.33 \\
\hline 10 & 1 & 0.68 & 95 & 1.38 & 0.80 & 0.30 \\
\hline 11 & 10 & 0.64 & 96 & 1.53 & 0.80 & 0.43 \\
\hline 12 & 10 & 0.63 & 98 & 1.32 & 0.78 & 0.25 \\
\hline \multicolumn{7}{|l|}{ Eustis } \\
\hline 1 & 10 & 1 & 100 & 1.40 & - & - \\
\hline 2 & 10 & 0.71 & 93 & 2.25 & 0.58 & 0.73 \\
\hline \multicolumn{7}{|l|}{ Vinton } \\
\hline 1 & 10 & 1 & 94 & 2.20 & - & - \\
\hline 2 & 10 & 0.68 & 99 & 3.30 & 0.24 & 0.56 \\
\hline \multicolumn{7}{|l|}{$Q D 2$} \\
\hline 1 & 0.01 & 1 & 93 & 1.04 & - & - \\
\hline 2 & 10 & 1 & 96 & 1.03 & - & - \\
\hline 3 & 0.01 & 0.81 & 93 & 1.16 & 0.62 & 0.1 \\
\hline 4 & 10 & 0.76 & 96 & 1.15 & 0.72 & 0.11 \\
\hline \multicolumn{7}{|l|}{$Q D 1$} \\
\hline 1 & 0.01 & 1 & 100 & 1.09 & - & - \\
\hline 2 & 10 & 1 & 100 & 1.08 & - & - \\
\hline
\end{tabular}

${ }^{a}$ Fraction of retention associated with adsorption at the air-water interface

$b_{\text {Retention due to adsorption at air-water interface }[\mathrm{AWIA}}=\mathrm{K}_{\mathrm{i}} \mathrm{A}_{\mathrm{aw}} / \theta_{\mathrm{W}}$ ] 